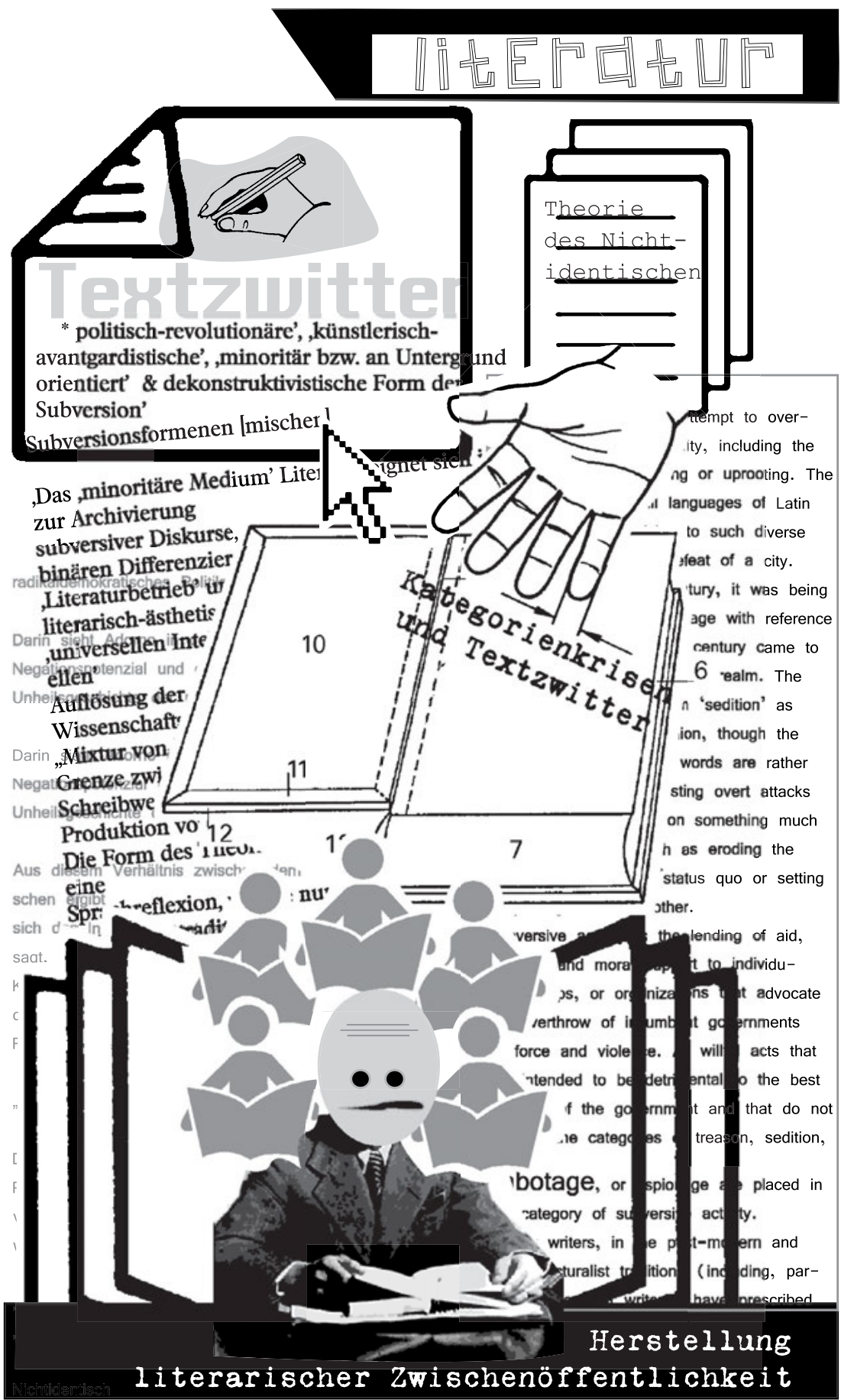

der Situation noch schlechter klarkommen als du. Redet darüber, daß es sinnvoll ist, ab sofort konsequent 


\title{
Textzwitter, Transvestitismus
}

\author{
und Terrorismus.
}

Subversive Konzepte in der

deutschsprachigen Gegenwartsprosa

\section{am Beispiel von Thomas Meineckes}

\section{Roman Tomboy}

THOMAS ERNST

»Die Macht der Benennung«, stellt Pierre Bourdieu fest, »ist von erheblicher Tragweite.« Und er ergänzt: »Worte können [...] Verheerungen anstellen« (Bourdieu I992: I65f.). Tatsächlich erheben sich heute aus der Summe flottierender Zeichen jene Worttürme der Macht, die ihre kleinen Erzählungen über >wissenschaftliche Erkenntnis<, >Gerechtigkeit des Marktes<, >die selbstbewusste Nation < und >Zweigeschlechtlichkeit zu hegemonialen Diskursen verfestigen. Benennungen, Worte und Sprache wurden vorrangig im Medium der Literatur verhandelt. Literatur sei heute jedoch, so Jochen Hörisch, angesichts der neuen und neueren Medien wie Fernsehen, Computer und Internet als Medium »minoritär« geworden: »Sie ist ein Anachronismus, ein langsames Medium, ein latenter Luxus, ein Relikt, ein schöner Überfluß. Doch eben auch ein Überfluß von bemerkenswerten Erkenntnis- und Einsichtsmöglichkeiten « (Hörisch I995: 38 ).

Wie weit reicht die Kraft der Literatur heute noch? Und in welcher Weise arbeitet sie sich an subversiven Konzepten ab? Wie lassen sich die vorherrschenden Worttürme ins Wanken bringen? Welche Sprachspiele eignen sich dazu, den kleinen Erzählungen und Geschichten ein alternatives Ende anzudichten?

Der vorliegende Beitrag will untersuchen, auf welchen Feldern und in welcher Weise die deutschsprachige Gegenwartsprosa heute noch >subversive Wirkungen< zu entwickeln versucht. Erstens wird auf einer allgemeinen Ebene beschrieben, auf welchen Feldern literarische Texte 
in der Gegenwart überhaupt noch subversive Wirkungen entfalten können. Zweitens wird - als ein Beispiel für einen subversiven Text auf der ästhetisch-literarischen Ebene - Thomas Meineckes Roman Tomboy (I998) einer exemplarischen Analyse unterzogen. ${ }^{\mathrm{I}}$ In einem Fazit wird drittens dargestellt, warum es sich auf dem literarischen Feld besser von >Aporien der Subversion< als von einer >Subversion der Subversion< sprechen lässt.

\section{Literarische Felder der Subversion}

Der Begriff der Subversion hat sich als geeignet erwiesen, um politische Kunst in der Gegenwart zu analysieren, hat jedoch vielfältige und widersprüchliche Bedeutungen angenommen. Dieser Beitrag geht davon aus, dass sich historisch vier Bedeutungsfelder des Subversionsbegriffs entwickelt und nebeneinander gestellt haben. Dabei handelt es sich um eine >politisch-revolutionäre <, eine >künstlerisch-avantgardistische<, eine >minoritäre bzw. an einem Untergrund orientierte s sowie eine >dekonstruktivistische Form $<$ der Subversion. ${ }^{2}$ Diese unterschiedlichen Konzepte werden, wie sich zeigen lässt, in Texten der deutschsprachigen Gegenwartsprosa aufgerufen, mit- und gegeneinander verhandelt und auf diese Weise archiviert.

Das minoritäre Medium Literatur eignet sich jedoch nicht nur zur Archivierung subversiver Diskurse, sondern inszeniert sich noch immer selbst als subversives Medium. Allerdings hat sich der Wirkungsbereich der Literatur, der sich einst auf das politisch-institutionelle, das medial-öffentliche und das literarisch-ästhetische Feld erstreckte, stark eingeengt. Literarische Texte sind jahrhundertelang durch Bücherverbrennungen und - seit Verbreitung des Buchdrucks - institutionalisierte Zensurapparate vernichtet und verboten worden, im deutschsprachigen Raum wurden während des Nationalsozialismus etwas mehr als I0.০०० Titel und in der DDR sogar insgesamt 30.000 Bücher verboten. ${ }^{3}$ In der demokratisch und kapitalistisch verfassten BRD weist die Liste der In-

1| Meineckes Roman Tomboy wird im Folgenden mit der Sigle TB abgekürzt.

2 | Vgl. die Ausführungen über die historisch gewachsenen Bedeutungsfelder des Begriffs der Subversion in der Einführung in diesen Band.

3| Aus diesem Grunde liegen zahlreiche literaturwissenschaftliche Untersuchungen vor, die die Subversivität von Literatur in der DDR durch den Akt der Zensur zu bestimmen versuchen. Vgl. u.a. Giovanopoulus 2000; Wüst I989. 
dizierungsstelle für jugendgefährdende Schriften aktuell allerdings nur "334 Bücher, Broschüren und Comics« (Löffler 2003: I7) aus. Über eine mögliche >subversive Kraft der deutschsprachigen Gegenwartsliteratur können die politischen Institutionen der BRD nur müde lächeln.

Gegen die marktwirtschaftliche Verfasstheit des Literaturmarktes und im Kampf um die mediale Öffentlichkeit hatte sich jedoch in den I96oer Jahren in der BRD ein alternatives Druck- und Vertriebsnetz ausgebildet. Abgesehen davon, dass viele Ziele dieser Untergrundliteratur nicht erreicht werden konnten, lassen sich im Nachhinein auch die binären Differenzierungen von >Untergrundliteratur< vs. >Literaturbetrieb < und >Unabhängigkeit< vs. > Mainstream < nicht mehr in dieser dichotomischen Form aufrecht erhalten. Zudem haben sich inzwischen andere Medien, wie vor allem das Internet, entwickelt, mit deren Hilfe eine alternative Öffentlichkeit viel einfacher zu etablieren ist ${ }^{4}$ - eine solche mit Hilfe des Mediums Literatur errichten zu wollen, erscheint heute als eine obsolete politische Strategie.

Es bleibt somit nur das literarisch-ästhetische Feld als Spezialdiskurs, auf dem Foucault ohnehin die eigentliche Wirkung politischer Literatur verortet, 5 und das heute keine breite gesellschaftliche Wir-

4 | Vgl. die Beiträge von Bernhard/Schäfer und Tangens in diesem Band. Allerdings gibt es auch heute noch unabhängige kleine (Untergrund-)Verlage und Editionen wie Alibri (Aschaffenburg), Blumenbar (München), Krash (Köln), KOOKbooks (Berlin/Idstein), Matthes Q Seitz (Berlin), Nautilus (Hamburg), Roadhouse (Hannover), Schwarze Risse/Assoziation A (Berlin/Hamburg), Tropen (Berlin), Trotzdem (Grafenau), Ventil (Mainz), Verbrecher (Berlin), Voland a Quist (Dresden) oder Zu Klampen (Springe), die sich allerdings mit vielfältigen Problemen konfrontiert sehen und zugleich den eigenen politischen Anspruch relativieren. Jörg Sundermeier vom Verbrecher Verlag verweist darauf, dass es heute nur noch »allerhöchstens dreißig« linke oder kleine Buchhandlungen gebe, während es zu den Hochzeiten der Studierendenbewegung, so >Veteran< Jörg Schröder vom März Verlag, »noch fünfhundert rote Buchhandlungen plus die Läden mit >Bücherstuben-Charakterく, die von >guten Buchhändlern geführt wurden« (Sundermeier und Schröder, zit.n. Plesch/Ullmaier 2004: 36), gegeben habe. Außerdem sei heute das Label >Untergrundliteratur von den großen Verlagen absorbiert worden, wie Lutz Schulenburg von der Edition Nautilus feststellt: »Was ist denn Underground? Das schreiben sich ja auch Kiepenheuer \& Witsch auf die Fahne« (Schulenburg, zit.n. Plesch/Ullmaier 2004: 37).

5 | Foucault verabschiedet schon 1972 den Typus des suniversellen Intellektuellen $<$ und plädiert für einen >spezifischen Intellektuellen es dem Intellektuellen [...] nicht mehr zu, sich an die Spitze oder an die Seite 
kung mehr erreicht. Während sich die Literatur zumindest in der ersten Hälfte des 20. Jahrhunderts noch als Leitmedium inszenierte, Bücher in autoritären und faschistischen Staaten massenhaft verboten wurden und mit der Figur des Intellektuellen eine literarische Ikone politischen Handelns präsent war, wurden seit den ig6oer Jahren der >Tod der Literatur< (vgl. Michel I968; Enzensberger I968), das >Grabmal des Intellektuellen ( vgl. Lyotard I985) und das >Ende der GutenbergGalaxis< (vgl. McLuhan I968; Bolz 1993) verkündet. Seither haben die »bildungsbürgerliche $>$ Mitte $<$ und mit ihr die traditionelle Bildungselite [...] schrittweise ihre kulturelle Hegemonie« verloren, so dass deren »kulturellen Werte und ästhetischen Standards [...] immer weniger als universalisierbar« (Bogdal 2004: 86) gelten können. Im Gegensatz dazu haben sich, so Klaus-Michael Bogdal, verschiedene Milieus mit unterschiedlichen Lesestilen und - daraus resultierend - verschiedene >Szeneliteraturen< entwickelt.

Für den deutschen Literaturmarkt heißt dies, dass als >subversiv $<\mathrm{zu}$ bezeichnende literarische Schriften nur von einer winzigen Minderheit überhaupt zur Kenntnis genommen werden: Die Satirezeitung Titanic hat eine Auflage von knapp I00.000 Exemplaren, Thomas Meineckes Roman Tomboy hat sich nach Aussage des Autors beim Suhrkamp Verlag ca. 15.00ofach verkauft, die bestverkauften Anthologien der Social Beat-Bewegung erreichten nur eine Auflage von I.००० Stück. Diese Literatur wird also nur von 0,I2 bis ०,०००I \% der 80 Millionen Bundesbürger/-innen überhaupt wahrgenommen.

Innerhalb des marginalisierten Spezialdiskurses >deutschsprachige Gegenwartsliteratur lassen sich jedoch noch immer verschiedene Richtungen unterscheiden, die sich auf dem literarisch-ästhetischen Feld um die Verhandlung subversiver Konzepte und ästhetischer Strategien bemühen. ${ }^{6}$ Im Folgenden wird sich dieser Aufsatz exemplarisch dem

aller zu stellen, um deren stumme Wahrheit auszusprechen. Vielmehr hat er dort gegen die Macht zu kämpfen, wo er gleichzeitig deren Objekt und deren Instrument ist [...]. Darum ist die Theorie [...] selbst eine Praxis« (Foucault, zit.n. Schlich 2000: 38 ).

6 | Dazu zählen die hermetische oder avancierte Literatur (Wolfgang Hilbig/I94I-2007, Elfriede Jelinek/*I946, Florian Neuner/*I972, Kathrin Röggla/*I97I), die experimentelle Popliteratur (Thomas Meinecke/*I955, Linus Volkmann/*I973), die Untergrundliteratur (Kersten Flenter/*I97I und die Social Beat-Bewegung, Bert Papenfuß/*I956), minoritäre Literaturen (Zafer Şenocak/*I96I, Yoko Tawada/*I960, Feridun Zaimoğlu/*I964, Raul Zelik/*I968), satirische Literatur (Eugen Egner/*I95I, Thomas Kapielski/*I95I, Jürgen Roth/*I968, Helge Schneider/*I955, Titanic), Science- 
Bereich der >experimentellen Popliteratur zuwenden und den Roman Tomboy von Thomas Meinecke analysieren. Den Hintergrund der Analyse bilden verschiedene poststrukturalistische Theorien, die im Roman selbst thematisiert werden. Nach einer sehr kurzen Einführung in den Roman werden anhand von drei ausgewählten Formmerkmalen und Inhalten des Textes dessen Verhandlung subversiver und ästhetischer Konzepte diskutiert: Zunächst die formale Ebene, auf der sich der Roman um die Auflösung der Grenze zwischen Wissenschaftssprache und Literatur bemüht (Textzwitter), anschließend die inhaltliche Ebene, auf der dekonstruktivistische Konzepte der Subversion (Transvestitismus) und politisch-revolutionäre Konzepte der Subversion (Terrorismus) miteinander verhandelt werden.

\section{Subversive Konzepte in Thomas Meineckes Roman Tomboy}

Thomas Meinecke wird von der Literaturkritik als ein Autor beschrieben, der an einer »Rückkehr zur Aufklärung« (Tuschick 1998) arbeite, sich dabei jedoch auf der Höhe poststrukturalistischer und postmoderner Theoriebildung befinde. Meinecke formuliere »die Hoffnung auf die Umgestaltung des autonomen Subjekts als handelndes zu einem Subjekt, das sich durch Erkenntnis definiert: Dass gerade die Bedeutungsüberschüsse für immer mehr Wahlmöglichkeiten sorgen und Widerstand ermöglichen« (Messmer 200I).

Tomboy spielt im Dreieck von Heidelberg, Ludwigshafen und Odenwald und berichtet vom Leben der Studentin Vivian Atkinson, die ihre Magisterarbeit über die misogynen Auslassungen Otto Weiningers in Form von Fragesätzen anfertigt, sich vorrangig in einem lesbischen, bi- und transsexuellen Milieu bewegt, das zudem ein hohes Interesse an politischen und feministischen Fragen hat. Der Roman bildet eine erzählte Zeit von Frühjahr 1997 bis Frühjahr I998 ab, in die - neben aktuellen Ereignissen wie einer Lesung von Judith Butler in München im Juni 1997 oder Lady Di's Tod Ende August 1997 - zahlreiche Reflexionen über verschiedene Probleme der Geschlechter(de) konstruktion eingebettet werden.

Fiction-Queer-Literatur (Nadja Sennewald/*I97I), Netzliteraturexperimente (Florian Cramer/*I969) und experimentelle oder postdramatische Theatertexte (René Pollesch/*I962, Falk Richter/*I969). Florian Neuners literarischer Beitrag Was tun wenn's brennt. Aussageverweigerung, Wahl der Mittel, der auch auf dieser Seite unten durchläuft, gibt einen guten Einblick in die shermetische oder avancierte Literatur und die Art und Weise, wie diese sich mit subversiven Konzepten auseinander setzt. 


\section{Textzwitter - die Auflösung der Grenze zwischen Wissenschaftssprache und Literatur}

Tomboy ist eine spezifische »Mixtur von Zitat und Narration« (Baßler 2002: I47), ein »Textzwitter aus Erzählung und theoretischer Spekulation« (Winkels I998), der die Grenze zwischen wissenschaftlicher und literarischer Sprache auflöst.7 Dies fällt dem Roman leicht, weil er sich primär auf wissenschaftliche Theorien bezieht, sie referiert, zitiert oder imitiert, die selbst der logozentrischen, nüchternen, traditionellen Wissenschaftssprache kritisch gegenüber stehen und demgegenüber auch in der Gestalt ihrer eigenen Sprache »ein allgemeines Plädoyer für die Differenz« (Münker/Roesler 2000: X) halten wollen.

Die wissenschaftliche Wissensproduktion befindet sich in einer Geltungskrise, wie Jean-François Lyotard in seinen Untersuchungen Das postmoderne Wissen. Ein Bericht (I979) und Der Widerstreit (I983) zu zeigen versucht hat: »Die Wissenschaft spielt ihr eigenes Spiel, sie kann die anderen Sprachspiele nicht legitimieren. [...] Vor allem aber kann sie sich auch nicht selbst legitimieren, wie es die Spekulation angenommen hatte« (Lyotard I994: II9). Diskursarten lieferten Regeln zur Verkettung ungleichartiger Sätze - es gebe jedoch keinen übergeordneten Diskurs, der als höchste Instanz über die richtige Verkettung von Sätzen bestimmen könnte. Genauso wie >die Wissenschaft< in verschiedene Fakultäten, die Fakultäten in verschiedene Fächer und die Fächer in verschiedene Schulen zersplittert sind, die untereinander in einem Widerstreit stehen, seien dies auch die einzelnen Sätze.

Als eine mögliche Konsequenz dieses Verlustes der Geltung wissenschaftlicher Sätze ließe sich die Möglichkeit denken, >wissenschaftli-

7 | Um nur ein Beispiel für die Mischung von narrativer und wissenschaftlicher Sprache zu nennen, sei hier die Einleitung in einen sexuellen Akt zwischen der bisexuellen Korinna Kohn und der >eigentlich Vivian Atkinson zitiert: »Keine Angst, Viv, sagte Korinna Kohn, als sie den Dildo aus ihrem Rucksack zog, dies hier ist auch ein Zeichen meiner persönlichen Akzeptanz heterosexueller Muster, nenne mich meinetwegen, mit Frauke Stöver, zwangsheterosexuell. Vivian Atkinson erinnerte sich, vor gar nicht allzu langer Zeit bei Judith Butler gelesen zu haben, mit dem sinnbildlich lesbischen Phallus werde das Verhältnis zwischen der Logik des ausgeschlossenen Widerspruchs und der Gesetzgebung der Zwangsheterosexualität auf der Ebene der symbolischen und körperlichen Morphogenese angefochten. [...] Also hob die Richterstochter ihre metallisch glänzende Purpur-Robe und schnallte sich das permanent erigierte, lehmfarbene Kunststoffglied um den nackten Körper« (TB 2I5). 
che < und >literarische< Sätze in einem Text zu vereinen, der die Grenze zwischen den beiden Sprech- und Schreibweisen aufhebt. Ganz im Sinne Lyotards verweist auch Meinecke darauf, dass sich die Unterschiede zwischen Literatur und Wissenschaft nivellieren: »Bei einigen Donna-Haraway-Sachen z.B. denke ich, das ist eher Science-Fiction im eigentlichen Sinne, also Wissenschaft als Fiktion. Andersherum handeln ja viele Theorien davon, dass selbst die Wissenschaften nichts anderes als eine narrative Erzählform sind « (Meinecke, zit.n. Brombach/Rüdenauer 1998). Zugleich begrüßt er ausdrücklich, dass er sich den wissenschaftlichen Anforderungen an seine Texte gegenüber verweigern kann, gerade weil sie eine spielerische Darstellung beinhalten: »Ich bin immer heilfroh, wenn ich keine wissenschaftliche Conclusio finden muß« (Meinecke, zit.n. Brombach/Rüdenauer 1998).

Damit verweigert sich Meinecke gegenüber den noch immer vorhandenen Geltungsansprüchen der wissenschaftlichen Sprache und ihrer Regelsysteme, die auf die Produktion von >Wahrheit< zielen. Er unterminiert in seiner Literatur diese Geltungsansprüche dadurch, dass er wissenschaftliche Theorien und Sprechweisen eingliedert in einen Erzählfluss, der sie gleichberechtigt neben Popmusikbezüge und populärkulturelle Zitate stellt und die von Wissenschaft (und teilweise auch Literatur) beanspruchte Hierarchie von Sprechweisen auflöst. Die Form des Theorie-Literatur-Textzwitters ermöglicht somit eine Sprachreflexion, wie sie gerade im Medium der Literatur möglich ist und die traditionellen >Wahrheitsideale des Wissenschaftsbetriebs und seiner Sprachregelungen problematisiert, parodiert und dekonstruiert.

Die Sprache des Romans unterminiert jedoch nicht nur die Geltungsansprüche der Wissenschaftssprache bzw. erweitert sie um neue Aussage- und Schreibweisen, vielmehr wird die (Alltags-) Sprache selbst zum Gegenstand des Romans. Rezensent/-innen und der Autor behaupten, dass Tomboy in einer >politisch korrekten Sprache< geschrieben sei: »So unsexy Dinge wie politisch korrekte Wortwahl sind mir schon was wert. Also hat Meinecke auch seinen Roman in freiwilliger Selbstkontrolle auf diskriminierende Passagen durchforstet und die weitverbreitete Usance, bei Frauen den Vornamen durch den bestimmten Artikel zu ersetzen (>die Irigaray<), rückgängig gemacht « (Nüchtern I998). ${ }^{8}$

8 | Meinecke stellt fest: »Man kann heute in der Tat nicht mehr so schreiben wie Anfang der Achtziger, aber auch nicht wie Mitte der Achtziger oder von mir aus auch wie vor I989. [...] In Mode a Verzweiflung gibt es zum Beispiel >den Neger< - das geht nun mal als Wort heute nicht mehr. Es mag lästig sein, aber da muss man eben >Afroamerikaner< schreiben « (Meinecke, zit.n. Büsser I998: I3I). 
In der Tat wird in Tomboy über die Schwierigkeit der Variation oder Dekonstruktion maskuliner und femininer Substantivendungen nachgedacht: als »Kellnerin, beziehungsweise Kellner« (TB 63) wird der/die Transvestit/-in Angela/o bezeichnet; das Binnen-I als Lösungsmöglichkeit wird thematisiert, aber nicht weiter reflektiert: »die drei FreundInnen, durch Hans mit großem Binnen-I« (TB 23I); Vivian verweist erstaunt darauf, dass »die Windows 95-Rechtschreibung doch tatsächlich vorschlug, den Terminus Frauenrechtler in Frauenrechtlerin zu verbessern« (TB I49) - also einen Akt auszuführen, der über die Korrektur der Sprache eine geschlechtliche Re-Identifizierung nach sich zöge; zudem sind die »Eigennamen [Luce Irigaray und Jacques Lacan, T.E.] und die Vokabel Phallogozentrismus [...] der Rechtschreibung von Windows 95 unbekannt« (TB 239).

Die >politische Korrektheit der Sprache und das akademische Interesse der Figuren in Tomboy verharren jedoch nicht in emanzipatorischem Ernst, denn die Fixierung der Figuren auf wissenschaftliche Theorien und eine exakte Sprache wird immer wieder Gegenstand einer gewissen Komik, indem die diffizilen theoretischen Ausführungen mit simplen Alltagsgeschehnissen kombiniert werden. ${ }^{9}$ Der biedere, analytische Ernst der traditionellen Wissenschaft wird also konterkariert - ganz im Sinne der eher spielerischen dekonstruktivistischen Verfahren, die den Protagonist/-innen selber viel Spaß machen und die vom Erzähler in einen ironischen, komischen Kontrast zu Alltagstätigkeiten gesetzt werden, wenn sie zu ausschweifend entwickelt werden.

\section{Transvestitismus - Gender Studies und Angela/o}

Der Roman spielt größtenteils in einer Frauen-Lesben-WG, deren Bewohnerinnen Vivian, Frauke, Ilse, Pat und Korinna sich intensiv mit den Gender Studies und also Theorien beschäftigen, die auf die Dekonstruktion der hegemonialen, binären Geschlechtermatrix abzielen. In Tomboy wird die Hierarchie der Geschlechter- und Sexualitätsmodelle umgedreht: Nimmt in der deutschen Gesellschaft

9 | Z.B. wird ein längeres Zitat aus einem Interview mit Pierre Bourdieu von Korinna kommentiert: »Frechheit, sagte Korinna Kohn, schnäuzte sich in ein Papiertaschentuch und trug das rote Bändchen wieder hoch. Brachte Batterien mit herunter, denn Heiners islamische Küchenuhr war stehengeblieben« (TB 237). Und das Streitgespräch über die Geschlechtsidentität Angela/os bringt auch die Umwelt ganz durcheinander: »Jedenfalls brachte der nervös gewordene Kellner die Rechnung, bevor die lärmende Runde auch nur annähernd aufgegessen hatte« (TB 9०). 
von 1996/97 (und auch noch heute) die binäre und heterosexuell codierte Geschlechtermatrix eine hegemoniale Position ein, während homo-, bisexuelle oder queere Lebensweisen minoritär sind, so wird in Tomboy eine ebensolche Gruppe aus hetero-, homo- und bisexuellen Frauen, einer/m Transvestitin/en (Angela/o Guida) sowie einem sich cross-dressenden Mann (Hans Mühlenkamm) ins Zentrum gesetzt, während Frauen, die emanzipatorischen Politikkonzepten oder dem Differenzfeminismus der I970er Jahre anhängen, sowie Männer, die patriarchale Verhaltensweisen zeigen, an den Rand gedrängt, karikiert und ausgeschlossen werden.

Die Konstruiertheit der Kategorie >Geschlecht< wird im Roman auf verschiedene Weisen vorgeführt: Die Figuren referieren und reflektieren die wichtigsten Grundannahmen und Streitpunkte der Gender Studies; ein patriarchaler Theorie-Diskurs, der ein misanthropes Frauen-Bild konstruiert, wird dargestellt und dekonstruiert (Vivian arbeitet sich in ihrer Magisterarbeit am Beispiel Otto Weiningers ab); durch Maskeraden, Cross-Dressing, vestimentäre Akte werden Geschlechter- und Sexualitätskategorien verschoben oder durchbrochen; der Transvestitismus wird als Unterminierung der binären Geschlechtermatrix vorgeführt (am Beispiel von Angela/o).

Dieser letzte Punkt soll - exemplarisch - ein wenig ausführlicher erläutert werden, denn an der Figur der/des Transvestitin/en Angela/o wird - parallel zur Auseinandersetzung der Protagonistinnen mit Jennie Livingstons Film Paris Is Burning ${ }^{10}$ - gezeigt, wie die binäre Geschlechtermatrix in der Praxis der Transvestiten subvertiert wird. Judith Butler setzt eine parodistische Praxis der Travestie und des Kleidertauschs einerseits, die nur »die Imitationsstruktur der Geschlechtsidentität« (Butler I99I: 202) offenbart, von einer weiter reichenden Praxis ab, die aufzeige, dass »das unvergängliche, geschlechtlich bestimmte Selbst durch wiederholte Akte strukturiert ist, die zwar versuchen, sich dem Ideal eines substanziellen Grundes der Identität anzunähern, aber in ihrer bedingten Diskontinuität, gerade die zeitliche und kontingente Grundlosigkeit dieses >Grundes< offenbaren « (Butler I99I: 207).

Diese Praxis beschreibt Marjorie Garber in ihrer Untersuchung Verhüllte Interessen. Transvestitismus und kulturelle Angst (1993), die in Tomboy an zahlreichen Stellen direkt oder indirekt zitiert wird, ${ }^{\text {II }}$ am Beispiel des Transvestitismus. Es sei »eine der konsistentesten und wirkungsvollsten Funktionen des Transvestiten in der Kultur [...],

10| Der Film Paris is Burning entstand zwischen I987 und I989 und dokumentiert Drag Balls von Latinos und Afro-Amerikanern in New York.

11 | Vgl. u.a. TB 8, 9, 87, I54. 
den Ort der [...] >Kategorienkrise< anzuzeigen « (Garber I993: 30), und diese >Kategorienkrise< ist nach Garber »die Grundlage von Kultur überhaupt« (Garber I993: 3I):

»Mit >Kategorienkrise< meine ich ein Misslingen von definitorischer Distinktion, eine Grenzlinie, die durchlässig wird und Grenzübertritte von einer (dem Anschein nach distinkten) Kategorie zu einer anderen erlaubt: schwarz/weiß, Jude/Christ, adlig/bürgerlich, Herr/Knecht, Herr/Sklave. Der Binarismus männlich/weiblich [...] wird im Transvestismus selbst in Frage gestellt oder ausgelöscht [...]. Das scheinbar spontane oder unerwartete oder zusätzliche Auftreten einer transvestischen Figur in einem Text [...], zeigt [...] eine Kategorienkrise an, einen unlösbaren Konflikt oder eine epistemologisch harte Nuss, welche der komfortablen Binarität die Festigkeit nimmt und das sich daraus ergebende Unbehagen auf eine Figur verschiebt, die bereits den Rand bewohnt, ja, die Marginalität inkarniert.« (Garber I993: 3I)

Garber versteht Kultur also als ein System von Binarismen, deren Konstruiertheit durch das Offenlegen ihrer notwendigen Begrenzungen deutlich wird. Auf dem Feld der Geschlechter-Binarität leistet dies die Figur des Transvestiten, die durch ihr Auftreten auf irgendeinem kulturellen Feld (Garber geht von einem weiten Textbegriff aus) die Begrenzungen der Binaritäten einerseits erst anzeigt, andererseits >verflüssigt<.

Die Figur der/s Transvestitin/en Angela/o Guida spielt eine zentrale Rolle in Tomboy, an ihr und mit ihr werden zahlreiche Grundannahmen des dekonstruktivistischen Feminismus diskutiert. Zugleich wird an ihr jedoch auch die Alltagspraxis einer Figur präsentiert, die die Konstruiertheit von Geschlechteridentitäten vorführt und exemplarisch die zahlreiche >Kategorienkrisen < des binären Musters >männlich/weiblichく auslöst. Bereits das Auftauchen Angela/os im Roman wird mit der Geschichte verbunden, dass sie/er einmal eine Veroner Go-Go-Tänzerin vertreten habe, was Vivian zu folgenden Fragen veranlasst: »Was hatten die Genossen dabei nun vor Augen geführt bekommen? Was hatten sie gesehen? Angelo? Eine Frau? Das Weibliche? Ließe sich, mit oder gegen Donna Haraway, behaupten, das geschätzte Publikum sei durch Angelas famosen Auftritt getäuscht oder gar betrogen worden? « (TB 58). Die Fragen werden nicht beantwortet, sondern eröffnen nur einen Verständnisrahmen, innerhalb dessen die Frage, welche Geschlechtlichkeit Angela/o zuschreibbar wäre, vertieft werden kann. Dass sich dafür im Sinne einer feststehenden Binarität der Kategorien >Mann und >Frau < keine klaren Lösungen finden lassen, zeigt an, dass die Figur der/s Angela/o der Geschlechter-Binarität ihre Festigkeit raubt. 
Vivian reflektiert die Kleiderwahl und Selbstzuschreibungen Angela/os und bemüht sich, die entsprechenden Wechsel nachzuvollziehen: Wenngleich »völlig flachbrüstig« (TB I5O), trage sie/er einen >weiblich k konnotierten Body, und folglich einen »Schwanz«, der »weiblichen Geschlechts war« (TB I5I). Später wandelt sich Angela/o vom Mann-Frau- zum Mann-Frau-Mann-Transvestiten: Frauke berichtet Vivian per Postkarte »von Angelas neuester Grille, der Male Impersonation [...]. Sie nennt sich an diesen Tagen Angelo, [...] sieht aus wie die Schriftstellerin Annemarie Schwarzenbach« - wobei sich Vivian fragt, ob diese >Männlichkeitsinszenierung $<$ nicht auch schon wieder gebrochen wird, »ob Angela feminine Dessous unter Angelos maskulinem Outfit trägt« (TB 205). Einige Zeit später vollzieht Angela/o wieder eine Female Impersonation, schlägt »ihre makellos rasierten Beine übereinander« und klatscht »entzückt in ihre manikürten Hände« (TB 243).

Die Figur Angela/os zeigt exemplarisch, wie Krisen der binären Kategorien >männlich/weiblich $<$ in einem literarischen Text erzeugt werden und die Festigkeit der Geschlechterbinaritäten subvertiert werden kann. Tomboy lässt sich entsprechend als ein Text lesen, der in diesem Sinne zahllose Kategorienkrisen erzeugt und somit das Konzept dekonstruktivistischer Subversion vorführt, reflektiert und archiviert.

\section{Terrorismus - eine Nebenerzählung}

In einer Art Nebenerzählung wird in Tomboy über die terroristischen Aktivitäten von Pat Meier, einer Außenseiterin der FrauenLesben-WG, und ihrem Nachbarn Bodo Petersen berichtet, ${ }^{12}$ deren Geschichte für Moritz Baßler eine »reale Subversion in der Tradition der Revolutionären Zellen« darstellt und die darum »einen dezenten, aber unüberhörbaren Kontrapunkt zur pastichehaften Archivierung des Gender-Diskurses « (Baßler 2002: 142) setze. Diese Nebenerzählung soll exemplarisch ausführlicher dargestellt werden, um zu verdeutlichen, dass Meineckes Roman neben dem Konzept der dekonstruktivistischen Subversion auch die politisch-revolutionäre Form der Subversion aufruft und diese Konzepte miteinander verhandelt. Es lässt sich also von einer indirekten Auseinandersetzung zwischen Transvestitismus und Terrorismus sprechen.

12 Die Geschichte von Pat Meier wird in TB 35, 70-75, 96-IO2, I45, I55-I57, I62f., 226 erzählt. 
Meier und Petersen gehören einer älteren Generation als die anderen Figuren an, Pat Meier ist Jahrgang I954 und hat sich dem Dekonstruktivismus gegenüber noch nicht geöffnet, verfolgt eher die materialistischen, linksradikalen und terroristischen Konzepte der I970er Jahre weiter. Sie »hatte oberhalb der Dossenheimer Steinbrüche einen sogenannten festen Ausguck installiert, im schwer zugänglichen Gestrüpp, von welchem aus sie überwiegend nachts, mit Hilfe diverser optischer Analysegeräte, die am westlichen Horizont wahrhaft gigantisch vor sich hin stinkende Badische Anilin- \& Soda-Fabrik, BASF, ausspionierte« (TB 35). Pat Meier bewegt sich in einer subversiven Szenerie: Das schwer zugängliche Gestrüpp, überwiegend nachts aufgesucht - als Ort; optische Analysegeräte - als Hilfsmittel zur Grenzüberschreitung; die $B A S F$ - als ein einem Drachen ähnlicher Feind (»gigantisch « und »stinkend«). Pat Meier, die äußerst kritisch zum Chemieriesen IG Farben und deren Nachfolgern Bayer, Hoechst AG und BASF steht (und eine »I972er Kampfschrift der RAF [...] an ihrem Busen trug«; TB 7I), lernt Vivians Nachbarn Bodo Petersen kennen, als dieser mit Vivian in jenem Steinbruch herumklettert, in dem zufällig auch Pats Unterstand steht. Petersen arbeitet ausgerechnet bei BASF und lobpreist anfangs sein Unternehmen für »all die zivilisatorischen Errungenschaften der chemischen Industrie« (TB 72). Doch schon bei diesem ersten Treffen kann Pat Meier Bodo Petersen für ihre Perspektive interessieren, indem sie ihn durch ihr Fernrohr blicken lässt - Petersen selbst ist unglücklich, dass es bereits I957 »aus betrieblichen Gründen, wie es hieß, werkpolizeilich untersagt worden« war, »Fotografien von seiner Arbeitsstätte anzufertigen« (TB 74).

Danach tauchen Pat Meier und Bodo Petersen gleichsam in den Untergrund des Erzählten ab, denn weder für die anderen Personen noch für den auktorialen Erzähler ist deutlich, wie sich das Verhältnis zwischen den beiden fortentwickelt: »War es eigentlich okay, daß er sich seinen geliebten Betrieb von einer politischen Extremistin madig machen ließ? Hatte er sich gar in Pat Meier verguckt? Beziehungsweise sie in ihn? Oder hielt sie, die Zielstrebige, ihn sich nur, um ihn, den Ahnungslosen, nach und nach zum Hochverräter umzuschulen? Waren denn seine heimlichen Fotos vom Steinbruch aus nicht bereits Werksspionage, ja Sabotage gewesen?« (TB IOI). Die Sache wird noch mysteriöser, als Vivian ihren Nachbarn Petersen und Pat Meier zusammen zu hören meint: »Vivian vernahm, synchron mit einem Hustenanfall Bodo Petersens, ein helles Lachen aus dem Treppenhaus. Sollte das etwa Pat Meiers Stimme sein? Die niemand in Handschuhsheim je hatte richtig lachen hören? Vivian schloß ihre Tür zum Flur« (TB I45f.). Pat Meier wird als eine verbiestert-schrullige Alt-Linke beschrieben, 
während Petersen offenbar schon (von ihr bzw. der von ihr verkörperten Revolution) >infiziert< worden ist.

Einige Zeit später sieht Vivian »in der Morgendämmerung«, wie Pat Meier »auf der ausgestorbenen Edinger Hauptstraße« aus Petersen Wohnung verschwindet, denn sie sieht just »im selben Moment Herrn Petersens Licht verlöschen. Komisch, wirklich komisch, amüsiert sich« Vivian (TB I55). Am nächsten Morgen klingelt Vivian bei Petersen, dieser trägt noch seinen Schlafanzug, der »auffallend verfleckt« sei, was Vivian sich fragen lässt: »Hatten die beiden Chemie-Fans im Bett mit irgendwelchen Säften herumexperimentiert?« (TB 156$)$.

Als Vivian Atkinson am Ende des Romans zu ihrem Wohnhaus zurückkehrt, sieht sie »Einsatzfahrzeuge der Polizei vor der Haustür« (TB 249) sowie, »oben angekommen, [...] Bodo Petersens aufgebrochene Wohnungstür; mehrere Kriminalbeamte durchsuchten seine Schränke, verwüsteten seine Einrichtung « (TB 249f.). Dabei finden die Beamten »einige alte Playboy-Magazine «, doch das ist ihnen »Nichts, meldete ein junger Polizist einem älteren und zuckte ratlos mit seinen Schultern, rein gar nichts « (TB 250) - was sowohl Petersen als auch die Polizisten außerhalb des dekonstruktivistischen Feminismus stellt, dem dieser Fund nicht >nichts< wäre. Überhaupt wird Bodo Petersen von Vivian als unangenehmer Typ gezeichnet, dem eine Reflexion der Geschlechterverhältnisse wie auch ein gepflegtes Äußeres ein Gräuel sind - ganz im Gegensatz zu Vivian, Frauke, Korinna und Hans, was das Konkurrenzverhältnis zwischen den unterschiedlichen politischen Konzepten der beiden Gruppen noch verstärkt. ${ }^{13}$

Das Buch endet mit einem neunzeiligen Absatz, der die >dekonstruktivistische Subversion< der Geschlechterkategorien der >politischrevolutionären Subversion < des Terrorismus gegenüber stellt: Einerseits sieht Vivian durch ihr Fenster, »wie die Polizeifahrzeuge in Edingens Hauptstraße einbogen«, zum Zwecke ihrer Vernehmung, während die terroristisch umtriebigen Bodo Petersen und Pat Meier schon inhaftiert sind. Zweitens verabreden sich Angela und Vivian, am morgigen Tag ins MS Connexion zum Tanzen - und schließen mit der entscheidenden Frage: »Was werden wir tragen? « (TB 25I)

Während also die politisch-revolutionäre, terroristische Subversion, die sich in direkte Opposition zu den Staatsorganen und Konzernen stellt, die Inhaftierung und das Verschwinden der Aktivist/-innen nicht in den Untergrund, sondern in die völlige staatliche Kontrolle zur Folge hat, kann die dekonstruktivistische Subversion ihre Spiele weiterspie-

13 | Die Geschichte von Bodo Petersen wird in TB 70-75, 96-IO2, I45f., I56f., I92, 250 erzählt. 
len - lustvoll, optimistisch, ungebrochen. Diese Kreuzung der beiden Subversionskonzepte steht an prominenter Stelle, am Ende des Buches, kann jedoch auf unterschiedliche Weisen gelesen werden: Als Abgesang auf die terroristischen Konzepte, die symbolisch zum Verschwinden gebracht werden; aber auch als Hinweis, dass die dekonstruktivistische Subversion zwar auf politische Effekte zielt, womöglich aber vor allem dem privaten Vergnügen dient.

\section{Von einer Subversion der Subversion über die affirmative Subversion zu den Aporien der Subversion}

Die Subversion der terroristischen Nebenerzählung durch die dekonstruktivistische Haupterzählung ist allerdings nicht alles - auch diese wird wieder subvertiert. Der Text wird von einer als männlich zu beschreibenden auktorialen Erzählfigur zusammengehalten, die einen >männlichen Blick auf das Erzählte wirft und den dekonstruktivistischen Enthusiasmus der Wohngemeinschaft ironisiert und problematisiert. Zudem enthält der Text zahlreiche ironische, parodistische und nonsenshafte Passagen, die ein mögliches didaktisches Verständnis des Textes, der eine Einübung der Gender Studies und ihrer Verfahren sein könnte, konterkarieren. Aus diesem Grunde wurde der Text auch von vielen Rezensent/-innen als Parodie auf die Gender Studies gelesen und von vielen Gruppen kritisiert. ${ }^{14}$ Der Roman Tomboy bietet jedoch beides: Er referiert, reflektiert und archiviert Positionen der dekonstruktivistischen Subversion; zugleich ironisiert und subvertiert er diese wieder und führt gerade in dieser gebrochenen Konstruktion politische Gehalte im Medium der Literatur vor.

Es erscheint jedoch unzutreffend, diese Verfahren als eine >Subversion der Subversion< zu bezeichnen, denn diese Denkfigur suggeriert eine problematische Temporalität: Einem (früher) als subversiv bezeichneten Akt, der diese Qualität heute verloren hat, müsse (jetzt) ein entgegengesetzter, konterrevolutionärer Akt der >Subversion der Subversion< folgen, der dann die >eigentliche Subversion< bedeuten würde. Diese Denkfigur beschreibt nur die inhaltliche Entleerung und formale Absorption einstmals subversiver oder avantgardistischer Akte, die durch politische, mediale oder gesellschaftliche Veränderungen ihre

14 | In seinem folgenden Roman Hellblau (200I) hat Meinecke folgerichtig auf eine auktoriale Erzählfigur verzichtet und diese auf drei ich-erzählende Figuren aufgesplittet. 
subversive Kraft verloren haben und nun eben als historisch obsolet >entlarvt< werden. ${ }^{\text {I5 }}$

Zutreffender lässt es sich im Bereich der dekonstruktivistischen Subversion von der >affirmativen Subversion< sprechen. Judith Butler beschreibt in ihrer Performanztheorie von Geschlecht, dass die Geschlechtsidentität als >Anschein einer inneren, festen Substanz<durch unzählige wiederholte performative Akte auf dem, im und um den Körper produziert wird. Entsprechend lasse sich eine solche Form >gezähmter Wiederholungen< (z.B. eines Machogehabes), die »als Instrumente der kulturellen Hegemonie « dienen, von »Formen parodistischer Wiederholung«, die »verstörend wirken« (Butler I99I: 204), unterscheiden. Diese störende Form dekonstruktivistischer Subversion, wie sie an der Figur Angela/o in Tomboy durchgespielt wird, realisiert ihre subversive Verschiebung also durch einen Akt der affirmativen Wiederholung, der jedoch durch Übertreibung oder Parodie eine Kategorienkrise auslöst, somit eine subversive Qualität gewinnt und durchaus im Kontext einer Subversion durch verschobene oder übertriebene Affirmation gelesen werden kann (vgl. auch Geier 2002).

Besonders plausibel erscheint die Rede von den >Aporien der Subversion< in der deutschsprachigen Gegenwartsliteratur, wie sie sich auch in Meineckes Roman zeigen. Subversive Konzepte werden präsentiert, archiviert, mit- und gegeneinander diskutiert, das eine subvertiert das andere. Die vorgeführten Konzepte werden wiederum von der Erzählerfigur ironisiert und parodiert - ein solcher literarischer Text erscheint als Komplex subversiver Konzepte, Motive und Figuren, die einander widersprechen und die Einnahme einer Position der >Wahrheit< oder die Präsentation einer >Lösung < unmöglich machen. Auf diese Weise gibt das minoritär gewordene Medium Literatur den Anspruch auf, engagiert, konkret, nachhaltig und direkt in die gesellschaftlichen Verhältnisse hinein zu intervenieren, und schreibt sich selbst die Rolle zu, subversive Konzepte und ästhetische Strategien zu archivieren, zu reflektieren und dabei jede Anmaßung einer neuen Utopie oder Wahrheit direkt wieder in Frage zu stellen.

Solange Worte noch immer Verheerungen anstellen, wird man auch Literaturen an der Frage bemessen können, ob sie >Sinn < oder >Un-Sinn< machen, eine >Lösung ob und wie sie die Worttürme der Macht zu unterbuddeln versuchen. Frei nach der situationistischen Gruppe Subversive Aktion steht auf dem Banner subversiver Literaturen: >Der Sinn der Bewegung ist ihr Scheitern< (vgl. Böckelmann/Nagel 2002). Wenn »die bedeutsamste

15 | Vgl. hierzu auch den Beitrag von Martin Doll in diesem Band. 
Neuerung « heutiger militanter Bewegungen, wie Antonio Negri und Michael Hardt behaupten, darin besteht, dass sie »die Tugenden aufrührerischen Handelns aus zwei Jahrhunderten subversiver Erfahrung« (Hardt/Negri 2003: 4I9) aufgreifen und neu verknüpfen, dann kann diese spezifische Form des minoritären und musealen Mediums Literatur einen kleinen Beitrag dazu leisten.

\section{Literatur}

Baßler, Moritz (2002): Der deutsche Pop-Roman. Die neuen Archivisten, München: Beck.

Böckelmann, Frank/Nagel, Herbert (2002) (Hg.): Subversive Aktion. Der Sinn der Organisation ist ihr Scheitern, Frankfurt a.M.: Neue Kritik.

Bogdal, Klaus-Michael (2004): »Deutschland sucht den Super-Autor. Über die Chancen der Gegenwartsliteratur in der Mediengesellschaft«. In: Clemens Kammler/Torsten Pflugmacher (Hg.), Deutschsprachige Gegenwartsliteratur seit I989. Zwischenbilanzen - Analysen - Vermittlungsperspektiven, Heidelberg: Synchron, S. 85-94.

Bolz, Norbert (I993): Am Ende der Gutenberg-Galaxis. Die neuen Kommunikationsverhältnisse, München: Fink.

Bourdieu, Pierre (1992): »Das intellektuelle Feld: Eine Welt für sich«. In: Pierre Bourdieu, Rede und Antwort, Frankfurt a.M.: Suhrkamp, S. I55-166.

Brombach, Charlotte/Rüdenauer, Ulrich (I998): »Gesampeltes Gedankenmaterial. Der Romancier Thomas Meinecke im Gespräch«. Frankfurter Rundschau, 2I.3.1998.

Büsser, Martin (I998): » Ich finde Musik eigentlich besser als Literatur<- Gespräch mit Thomas Meinecke«. testcard. Beiträge zur Popgeschichte, Heft 6/1998, Mainz: Ventil, S. I30-I35.

Butler, Judith (I99I): Das Unbehagen der Geschlechter. Frankfurt a.M.: Suhrkamp.

Enzensberger, Hans Magnus (I968): »Gemeinplätze, die Neueste Literatur betreffend«. In: Kursbuch 15, Frankfurt a.M.: Suhrkamp, S. I87-I97.

Garber, Marjorie (I993): Verhüllte Interessen. Transvestitismus und kulturelle Angst, Frankfurt a.M.: Fischer.

Geier, Andrea (2002): »Weiterschreiben, Überschreiben, Zerschreiben. Affirmation in Dramen- und Prosatexten von Elfriede Jelinek und Marlene Streeruwitz«. In: Ilse Nagelschmidt/Alexandra Hanke/Lea Müller-Dannhausen/Melani Schröter (Hg.), Zwischen Trivialität und Postmoderne. Literatur von Frauen in den goer Jahren, Frankfurt a.M. u.a.: Lang, S. $223-246$.

Giovanopoulus, Anna-Christina (2000): Die amerikanische Literatur in der 
DDR. Die Institutionalisierung von Sinn zwischen Affirmation und Subversion, Essen: Die Blaue Eule.

Hörisch, Jochen (I995): »Verdienst und Vergehen der Gegenwartsliteratur«. In: Christian Döring (Hg.), Deutschsprachige Gegenwartsliteratur. Wider ihre Verächter, Frankfurt a.M.: Suhrkamp, S. 30-48.

Löffler, Sigrid (2003): ">Ich übergebe der Flamme die Schriften von...< Feuer und Verbote in der Bücherwelt. Ein Streifzug aus Anlass der deutschen Bücherverbrennungen vor siebzig Jahren, am Io. Mai I933«. In: Literaturen, Heft 5 (2003), S. 6-I7.

Lyotard, Jean-François (I985): Grabmal des Intellektuellen, Graz/Wien/Böhlau: Passagen.

Lyotard, Jean-François (I994): Das postmoderne Wissen. Ein Bericht, Wien: Passagen (3. Aufl.).

McLuhan, Marshall (I968): Die Gutenberg-Galaxis. Das Ende des Buchzeitalters, Düsseldorf/Wien: Econ.

Meinecke, Thomas (I998): Tomboy. Roman, Frankfurt a.M.: Suhrkamp.

Meinecke, Thomas (200I): Hellblau. Roman, Frankfurt a.M.: Suhrkamp.

Messmer, Susanne (200I): »Eine elegante Einladung zum Dialog. Thomas Meinecke schreibt an seinem Projekt der postkolonialen Literatur weiter«. Die Tageszeitung, 03.09.200I.

Michel, Karl Markus (I968): »Ein Kranz für die Literatur«. Kursbuch I5, Frankfurt a.M.: Suhrkamp, S. 169-186.

Münker, Stefan/Roesler, Alexander (2000): Poststrukturalismus, Stuttgart; Weimar: Metzler.

Nüchtern, Klaus (I998): »DJ Gender Studies«. In: Falter, Heft 39 (I998).

Plesch, Tine/Ullmaier, Johannes u.a. (2004): »Linke Verlage in Deutschland. Eine Gesprächsrunde«. In: Martin Büsser/Roger Behrens/Jens Neumann/ Tine Plesch/Johannes Ullmaier (Hg.), Linke Mythen. testcard. Beiträge zur Popgeschichte, Heft I2 (2004), Mainz: Ventil, S. 32-39.

Schlich, Jutta (2000): »Geschichte(n) des Begriffs >Intellektuelle««. In: Jutta Schlich (Hg.), Intellektuelle im 20. Jahrhundert in Deutschland. Ein Forschungsreferat. Internationales Archiv für Sozialgeschichte der deutschen Literatur. II. Sonderheft, Tübingen: Max Niemeyer, S. I-II3.

Tuschick, Jamal (I998): »Mehr Groove im Diskurs. DJ und Schriftsteller Thomas Meinecke im Literaturhaus«. Frankfurter Rundschau, o8.12.1998.

Winkels, Hubert (1998): »Was ist Sache, Mann? Die Frau. Thomas Meinecke betreibt den Geschlechterdiskurs in Romanform«. Die Zeit, o8.Io.I998.

Wüst, Karl Heinz (1989): Sklavensprache. Subversive Schreibweisen in der Lyrik der DDR I96I-I976, Frankfurt a.M. u.a.: Lang. 


\title{
Textzwitter, Transvestitismus und Terrorismus
}

\author{
Ernst, Thomas
}

Dieser Text wird über DuEPublico, dem Dokumenten- und Publikationsserver der Universität Duisburg-Essen, zur Verfügung gestellt.

Die hier veröffentlichte Version der E-Publikation kann von einer eventuell ebenfalls veröffentlichten Verlagsversion abweichen.

DOI: https://doi.org/10.14361/9783839406779-006

URN: urn:nbn:de:hbz:464-20190318-170540-5

Link: https://duepublico.uni-duisburg-essen.de:443/servlets/DocumentServlet?id=48384

Rechtliche Vermerke:

Thomas Ernst dankt dem Walter de Gruyter Verlag für die freundliche Genehmigung, diesen Text in der Verlagsversion online veröffentlichen zu dürfen.

Quelle: Erschienen in: Thomas Ernst, Patricia Gozalbez Cantó, Sebastian Richter, Nadja Sennewald, Julia Tieke (Hg.): SUBversionen. Zum Verhältnis von Politik und Ästhetik in der Gegenwart. Bielefeld: transcript, 2008, S. 109-128. 